\title{
The Effect of Sample Preparation on Observed Microstructure in Polymeric and Polymer Composite Gas Separation Membranes
}

\author{
Charles J. Holt ${ }^{1}$, Kenneth J. Balkus, Jr. ${ }^{1}$, John P. Ferraris ${ }^{1}$, and Inga H. Musselman ${ }^{1}$ \\ 1. Department of Chemistry and Biochemistry, The University of Texas at Dallas, Richardson, USA.
}

Leeuwenhoek, one of the first microscopists, understood that the interpretation of features in a magnified image depends largely on the quality of the prepared sample. Today, one of the most utilized microscopes is the scanning electron microscope (SEM) due to its high throughput, ease of operation, and simple sample preparation. While there has been extensive research on sample coatings for SEM imaging (e.g., conductive metals, coating thickness, charge effects) [1], less attention has been given to artifacts introduced from the fracture and cutting of samples for high magnification imaging. In the field of gas separations, liquid nitrogen is commonly used to "freeze-fracture" polymer membranes to visualize internal microstructure. While this method allows for high throughput, it also imparts artificial morphology and topology that can easily be misinterpreted [2]. In this paper, SEM and atomic force microscopy (AFM) imaging techniques were utilized to showcase the importance of microtomy for gas separation membrane research.

A variety of polymers and additives were selected to represent the numerous materials and common internal morphologies present in membranes for gas separation research. Polybenzimidazole (PBI) was purchased from PBI Performance Products, Inc. $\left(\mathrm{M}_{\mathrm{w}} \sim 30 \mathrm{KDa}, 26 \mathrm{wt} \%\right.$ in dimethylacetamide with 1.5\% (w/w) LiCl). Matrimid $5218^{\circledR}$ was obtained from Ciba Specialty Chemicals (Huntsman Advance Materials Americas, Inc.). Polysulfone (PSf) was acquired from Solvay Chemicals, Inc. (Udel ${ }^{\circledR}$ P-3500 LCD). Cellulose acetate $\left(\mathrm{M}_{\mathrm{w}} \sim 100 \mathrm{KDa}\right)$ was purchased from Acros Organics (Fisher Scientific). Basolite $^{\circledR}$ Z1200 (ZIF-8) was purchased from Aldrich. The co-polyimide 6FDA-DAM:DABA (3:2) (6FDD) and colloidal ZIF-8 (cZIF-8) were synthesized according to published procedures [3-4]. Polymer, polymer blend, and mixed-matrix membranes (MMM) were fabricated and annealed using our custom protocol [3]. Cross-sections of membranes were prepared via freeze-fracture using liquid nitrogen and by microtoming. Membranes were submerged in liquid nitrogen for several seconds then pressed against the bottom of the low-form nitrogen dewar flask to fracture the membrane and reveal a cross-section. A Leica EM UC7 Ultramicrotome was used to cut the membrane samples with either $45^{\circ}$ glass knives or a $45^{\circ}$ diamond knife supplied by Diatome. The freeze-fractured and microtomed samples were imaged by SEM (Zeiss Supra 40) and AFM (Bruker Multimode 8 with "J" scanner in PFQNM) to compare their microstructures.

The most common features observed in membrane cross-sections prepared by freeze-fracture are stretched or elongated deformations, craters, flakes, and web-like structures (Figure 1). In MMMs or polymer blend membranes, interfacial voids are typically amplified or introduced during freeze-fracture due to the disruptive force applied during the preparation. It is also difficult to determine the level of miscibility of blends and wetting of additives by the polymer matrix using samples prepared by freezefracture. An example of the difference in cross-sections prepared by freeze-fracture and microtoming is shown in Figure 2. The rough topography in the polymer blend is created from the freeze-fracture process (Figure 2A) and shows voids between the dispersed and continuous phases, leading to the conclusion that poor compatibility exists between the two polymers. In contrast, a clean and smooth 
cross-section created by microtoming the membrane (Figure 2B) shows the polymers actually have some degree of compatibility since no large voids are present between the domains.

Utilizing microtomy as a sample preparation technique for microscopy is common practice in several analytical disciplines. In gas separation membrane research, however, freeze-fracture is still the most common sample preparation technique for imaging. While no group relies solely on microscopy to characterize the membranes, it is still critical to understand the effect that sample preparation has on the observed internal morphology. Sample preparation using microtomy results in a more accurate representation of the internal microstructure of gas separation membranes and allows for more rigorous characterization using other microscopy techniques. For example, the study of polymer blend interfaces is made possible by using a microtome to prepare membrane cross-sections (Figure 3) [5].

\section{References:}

[1] Schossig-Tiedemann, M., Paul, D., Journal of Membrane Science 187 (1) (2001), p. 85.

[2] Sawyer, L., Grubb, D. and Meyers, G. in "Polymer Microscopy", (Springer-Verlag, New York) p. 489.

[3] Panapitya, N.P. et al, Applied Material Interfaces 7 (33) (2015), p. 18618.

[4] Panapitya, N.P. et al, Polymer, 55 (8) (2014), p. 2028.

[5] The authors acknowledge funding from the National Science Foundataion CBET-1403950.

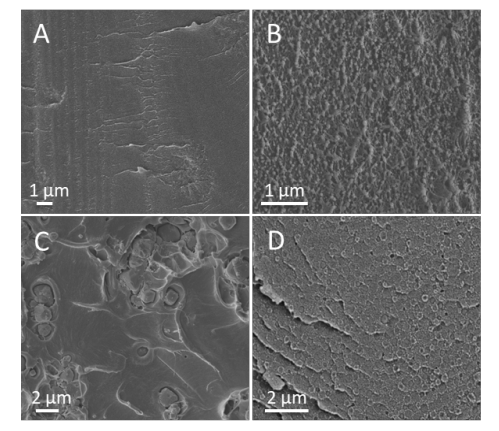

Figure 1. SEM images of cross-sections of gas separation membranes prepared by freezefracture. (A) PBI, (B) 40 wt $\%$ MOP-18/Matrimid $5218^{\circledR}$ MMM, (C) $60 \mathrm{wt} \%$ Zeolite-A/PSf MMM, and (D) 50/50 PBI/6FDD blend.

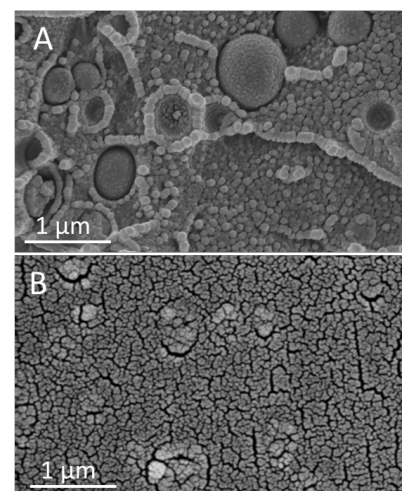

Figure 2. SEM images of a 90:10 PBI/6FDD blend prepared by (A) freeze-fracture and (B) ultramicrotome.

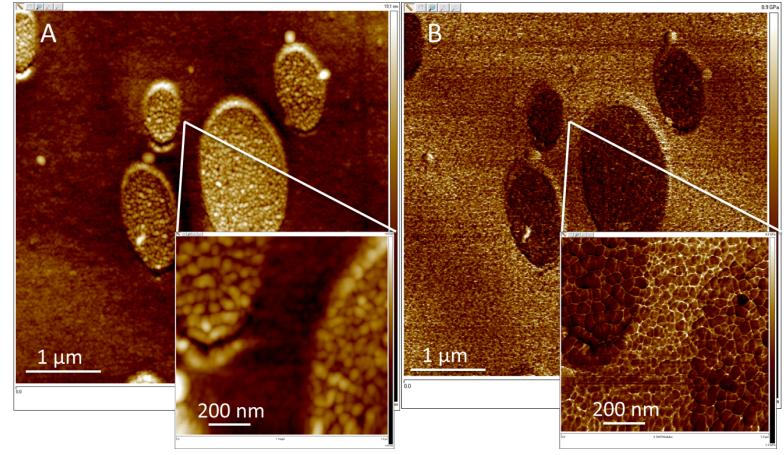

Figure 3. AFM (A) height and (B) modulus images of a 50:50 PBI/6FDD blend prepared by ultramicrotome. 